\title{
Volumetric Proper Orthogonal Decomposition of an impinging jet using SPIV measurement
}

\author{
Jana Hamdi ${ }^{1,}{ }^{*}$, Kamel Abed-Meraïm ${ }^{1}$, Hassan Assoum ${ }^{2}$, Anas Sakout ${ }^{1}$, Tarek Mrach $^{1}$, \\ Marwan Al-Kheir ${ }^{1}$, Laurent Rambault ${ }^{3}$, Sebastien Cauet $^{3}$ and Eric Etien ${ }^{3}$ \\ ${ }^{1}$ LaSIE, University of La Rochelle, Avenue Michel Crépeau, Pôle Sciences et Technologies, 17000 \\ La Rochelle, France \\ ${ }^{2}$ Beirut Arab University - Tripoli Campus, Corniche El Meena, Tripoli, Lebanon \\ ${ }^{3}$ Lias -Ensip, Univeristé de Poitiers, 2 rue Pierre Brousse, TSA 41105, 86073 Poitiers Cedex 9 - \\ France
}

\begin{abstract}
Impinging jets which interact with sharp edges like slotted plates can be found in ventilation and air conditioning systems. In some cases, these configurations can be a source of desirable sounds like in musical instruments. However, they can be a source of noise in other applications such as air conditioning systems. Thus in order to reduce the noise produced from such configurations a more profound study of the flow dynamics should be implemented. All previous studies of impinging jets on a slotted plate were done in 2D. Accordingly, the objective of the present study was to reconstruct the studied volume by combining stereoscopic PIV measurements and the POD method. Consequently, an experimental set up has been developed and implemented in order to generate a flow that hits a slotted plate. On the other side, the PIV technique was used in order to study the dynamics of such a flow. The kinematic fields of twenty two parallel planes are measured by means of stereoscopic PIV. Afterwards, the analysis of the most energetic modes was obtained by the application of the snapshot POD on the kinematic fields obtained by POD. The results showed that the reconstruction of each of the planes needed only three first three modes. The reconstructed volume is obtained by an interpolation of the reconstructed planes which were phased by a trigger signal. The analysis of the vortex structures were done by the application of several vortex detection criteria.
\end{abstract}

\section{Introduction}

One of the important subject in the scientific research is Turbulence which is one of the subjects the least understood [1]. In this context, i.e. for turbulent jets of high Reynolds number Navier-Stokes equations which are non-linear are difficult to be resolved even for simple geometries. Consequently, this constraint imposes difficulties to obtain a good approximation of the solutions of complex problems. Thus a new ideology of the model reduction was imposed. One of the best-known methods is the proper orthogonal

\footnotetext{
*Corresponding author: jana.hamdi@univ-lr.fr
} 
decomposition (POD) which aims to find the first dominant eigenvectors of the spatial correlation of the velocity field. Lumley first introduced the Proper Orthogonal Decomposition in the context of turbulence. Another approach of this decomposition was proposed by Sirovich [2], it's called the snapshot POD and it the exact symmetry of the classical POD. An example of application of this method is [3], where Druault applied it as a method of time interpolation from PIV data.

In this study, a high speed plane jet impinging on a slotted plate at $\mathrm{Re}=4458$ was considered. Obviously, the flow presents a three dimensional character that should be analysed. A non-volumetric nor a simultaneous method was then introduced in this study: an interpolation of parallel planes. Twenty-two parallel planes were measured by SPIV technique. The snapshot POD was applied to the results in order to reconstruct the flow with the minimum amount of modes. Note that the jet was measured for a confinement of $\mathrm{L} / \mathrm{H}=4$ and a Reynolds number of 4458 which is a configuration corresponding to a noisy and turbulent set up as shown by Assoum [4].

\section{Experimental Setup}

The kinematic field of twenty two transversal parallel planes are measured using the stereoscopic PIV technique. As the PIV technique is based on acquisitions of the illuminated flow, both the cameras and the optical fiber generator of the laser plane were fixed on a displacement system. Initially, the displacement system is fixed at the middle plane of the plate which was named $\mathrm{P}_{0}$. This displacement system was then moved forward twenty two times in order to illuminate and capture images of the planes of study. Note that the twenty two measured kinematic fields were $2 \mathrm{~mm}$ apart from one another.

\section{Results}

The kinematic field of the twenty two parallel planes were obtained by an adaptive multigrid algorithm cross-correlation of the two images recorded by the cameras. The snapshot POD was applied on each of the measured planes separately. That is the correlation tensor was built for each plane aside. The POD analysis has shown that for all the planes the first three modes are the most energetic. The reconstructed planes with only the first three modes has shown a phase shift since the measurements were done independently (see Fig. 1(a)). Consequently, a trigger signal was used as tool for plane synchronization. This technique allowed us to establish the volume of study as shown in Fig. 1(b).

(a)

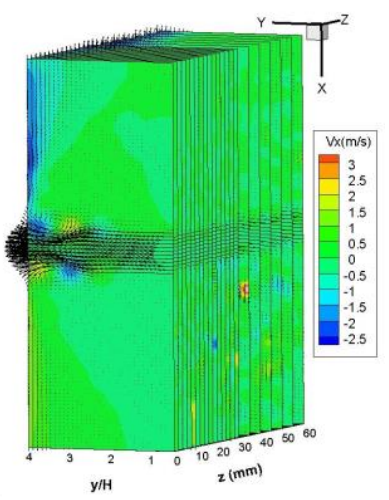

(b)

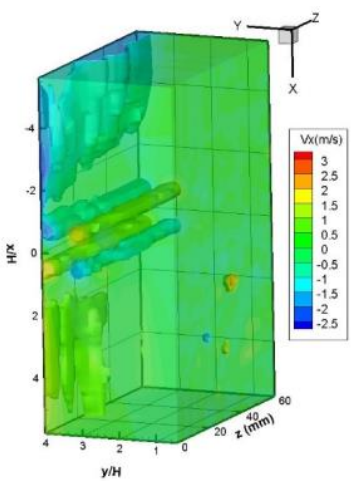

Fig. 1. (a) Reconstructed planes by POD, (b) Reconstructed Volume by Snapshot POD 
The use of the snapshot POD is due to the fact that this method is a model reduction method that compress information (with three modes and all temporal coefficients, it is possible to reconstruct all the data). This interpolation is carried out on the reconstructed planes by an inverse distance weighting method. A homogeneous data volume is then obtained. Several vortex detection criteria were applied to detect the vortex trajectories and centres. In the present study, we chose to present only the swirl iso-surfaces coloured of the transversal velocity (see Fig. 1). The results show the vortex rolls which appear downstream the jet close to the nozzle of the plate. In addition to the vortex rolls, the results show shear layers on the sides of the plate.

\section{Conclusions}

The 3D reconstruction of an impinging jet on a slotted plate by combining POD and SPIV measurements was the objective of the present work. First, the kinematic field of twentytwo parallel planes was calculated by means of stereoscopic PIV. Since the data is time resolved the snapshot POD was applied to the SPIV measurements of each of the parallel planes. A reference trigger signal was then considered in order to synchronise the planes which were measured independently. Afterwards, an interpolation of the synchronized reconstructed planes has led us to a reconstructed volume. Thus by using only three modes, i.e. small amount of information, the volume of study is obtained by a reconstruction of 2D$3 \mathrm{C}$ measurements.

\section{Acknowledgments}

This work would not have been possible without the financial support of Nouvelle Aquitaine region.

\section{References}

[1] G Berkooz, P Holmes, et and J. L. Lumley, « The Proper Orthogonal Decomposition in the Analysis of Turbulent Flows », Annu. Rev. Fluid Mech., vol. 25, nº 1, p. 539-575, 1993.

[2] L. Sirovich, «Turbulence and Dynamics of Coherent Structures Part I: Coherent Structures », $Q$. Appl. Math., vol. 45, no 3, p. 561-571, 1987.

[3] P. Druault, P. Guibert, et F. Alizon, « Use of proper orthogonal decomposition for time interpolation from PIV data », Exp. Fluids, vol. 39, nº 6, p. 1009-1023, sept. 2005.

[4] H. H. Assoum, M. El Hassan, K. Abed-Meraim, et A. Sakout, " The vortex dynamics and the self sustained tones in a plane jet impinging on a slotted plate », Eur. J. Mech. - BFluids, vol. 48, p. 231-235, nov. 2014. 